\title{
Addition of methylphenidate to intensive dialectical behaviour therapy for patients suffering from comorbid borderline personality disorder and ADHD: a naturalistic study
}

\author{
Paco Prada - Rosetta Nicastro · Julien Zimmermann • \\ Roland Hasler • Jean-Michel Aubry • \\ Nader Perroud
}

Received: 25 July 2014 / Accepted: 11 January 2015/Published online: 30 January 2015

(C) Springer-Verlag Wien 2015

\begin{abstract}
Attention deficit hyperactivity disorder (ADHD) is frequently comorbid with borderline personality disorder (BPD). However, few studies have examined how comorbid BPD-ADHD patients, treated or not with methylphenidate (MPH), respond to psychotherapy compared to non-comorbid BPD patients. In this perspective, we used a naturalistic study to compare, during a monthlong intensive dialectical behaviour therapy (DBT), the clinical course of BPD patients and comorbid BPD-ADHD patients who were treated or untreated with MPH. Out of the 158 BPD patients recruited, 59 had adult ADHD as a comorbidity; among these, 29 underwent a treatment with MPH or des-methylphenidate, while the 30 others did not. MPH treatment was given non-randomly and only when ADHD was considered to be hampering the capacity of the subjects to follow the therapy. Patients completed the following forms upon admission and after 1 month of treatment: the adult ADHD Self-Report Scale (ASRS v.1.1), the Barratt Impulsiveness Scale (BIS-10), the State-Trait Anger Expression (STAXI), the Beck Depression Inventory II (BDI-II), and the Beck Hopelessness Scale. At
\end{abstract}

P. Prada $\cdot$ R. Nicastro $\cdot$ J. Zimmermann $\cdot$ N. Perroud $(\bowtie)$ TRE Program, Service of Psychiatric Specialties, Department of Mental Health and Psychiatry, University Hospital of Geneva, 20 rue de Lausanne, 1201 Geneva, Switzerland e-mail: nader.perroud@hcuge.ch

P. Prada

e-mail: paco.prada@hcuge.ch

R. Hasler · J.-M. Aubry $\cdot$ N. Perroud

Department of Psychiatry, University of Geneva, Geneva,

Switzerland

J.-M. Aubry

Bipolar Disorder Unit, Department of Mental Health and

Psychiatry, University Hospital of Geneva, Geneva, Switzerland baseline, comorbid BPD-ADHD patients showed significantly higher impulsiveness than BPD patients. In the entire sample, there was a significant decrease in all dimensions ranging from small to large effect sizes during the 4-week intensive DBT. BPD-ADHD patients who were undergoing MPH treatment showed a significantly improved response to DBT treatment for Trait-State Anger scores, motor impulsiveness, depression severity, and ADHD severity, when compared to those without stimulant medication. This study outlines the importance of systematically screening BPD patients for ADHD, since a MPH-based treatment will improve the symptoms of patients who are comorbid for BPD and ADHD. Due to the non-random allocation of subjects, more severely affected patients were more readily placed on MPH; this suggests that the more severe the ADHD symptoms, the greater the chance for the patient of being treated.

Keywords ADHD - Borderline personality disorder . Methylphenidate $\cdot$ Response to treatment $\cdot$ Psychiatry

\section{Introduction}

The treatment of borderline personality disorder (BPD) is complex and challenging for many reasons, among which features its high frequency; BPD affects roughly $2 \%$ of the general population and up to $15 \%$ of patients undergoing psychiatric treatment or consultations (Skodol et al. 2002). Also, some of the core elements of the disorder, such as impulsiveness (Herpertz et al. 1997), emotion management issues (in particular anger) (Herpertz et al. 1999), selfharm, and repeated suicide attempts, cause significant mortality and morbidity rates (Lieb et al. 2004). A dramatic consequence of those behavioural difficulties is that they 
generate many interruptions of treatment that hinder efforts to properly monitor the patient's clinical course within the mental health system (Lieb et al. 2004). The high frequency of comorbidities is another distinguishing feature of the disorder that complicates the patient's clinical profile (Zanarini et al. 1998; Skodol et al. 2002).

It is currently an accepted fact that psychotherapy is the first-line treatment of BPD, and several BPD-specific interventions have been developed and studied; various therapies have now been shown to be effective in treating this disorder (Stoffers et al. 2012), including, among others, the dialectical behavioural therapy (DBT) (Lynch et al. 2007), the mentalisation-based therapy (MBT) (Bateman and Fonagy 2010), or the transference-focused therapy (TFP) (Yeomans et al. 2013). Pharmacological treatment is used as a complement to psychotherapy as it partially targets only some of the dimensions of the disorder, such as impulsive behavioural dyscontrol and affective dysregulation (Ingenhoven et al. 2010). In the complex pharmacological treatment algorithm of BPD (Ingenhoven et al. 2010), related comorbidities should be included and targeted (American Psychiatric Association 2001).

Among the comorbidities frequently encountered in BPD sufferers, the adult attention deficit hyperactivity disorder (ADHD) is of particular interest, because of its comorbidity rate of about $16 \%$ on the one hand (Philipsen et al. 2008), and because it appears to share some core features with BPD, such as impulsiveness, emotional dysregulation, anger and stress management issues (Philipsen et al. 2009; van Dijk et al. 2012) on the other. Difficulty to control emotions and mood lability are indeed recognised as core symptoms of ADHD since the Wender Utah criteria were published, although they have not been recognised by the DSM-V. Several recent papers support the idea that emotion regulation difficulties play a central role in ADHD (Matthies et al. 2014; Shaw et al. 2014). Moreover, both BPD and ADHD share the same developmental aspects (Zanarini et al. 1997; Zanarini 2000; Fossati et al. 2002).

Studies show that despite their resemblance, these disorders are in fact distinct (Davids and Gastpar 2005; Philipsen 2006); ADHD differs from BPD mainly because it relies on inhibitory deficit (Nigg 2001; Lampe et al. 2007; Jacob et al. 2010). Furthermore, we previously compared aspects such as impulsiveness and anger among patients suffering from BPD, ADHD, and comorbid patients affected by both disorders, with the latter group showing higher aggression and substance abuse rates (Prada et al. 2014). It has been suggested that ADHD could be an aggravating factor when comorbid with BPD (Philipsen et al. 2008). There are only very few studies, which are mainly case studies, about the treatment of comorbid BPD-ADHD patients. Those studies showed that a proper treatment for ADHD improves the patient's symptoms (van Reekum and
Links 1994; Golubchik et al. 2008). It is also interesting to note that the treatment of ADHD has been shown to improve emotion dysregulation in ADHD patients (Shaw et al. 2014), and it might also be useful to BPD patients.

The aim of this study is to compare the clinical course of BPD patients and comorbid BPD-ADHD patients, treated or untreated with methylphenidate (MPH) compounds during a month-long therapy program. It is a specialised treatment for the DBT type of the BPD disorder, the efficiency of which has recently been demonstrated (Perroud et al. 2010b). The current investigation is part of a broader study aiming at deciphering clinical and dimensional aspects of BPD, ADHD, and comorbid BPD-ADHD patients (Prada et al. 2014). The question that we seek to answer here is whether elements exist to differentiate these three groups of patients (BPD; BPD-ADHD treated; and BPD-ADHD untreated), at the beginning and at the end of the treatment. We also wish to examine the relevance of using a concomitant treatment for ADHD on BPD patients suffering from this comorbidity. To the best of our knowledge, this is the first study to examine the response to combined psychotherapy and pharmacotherapy in a sample of BPD and comorbid BPD-ADHD subjects.

\section{Methods}

This is a naturalistic study conducted in an outpatient psychotherapy centre specialised in treating BPD sufferers and adult ADHD sufferers. Our centre provides DBT, following the model developed by Linehan (1993). The treatment program is specific in that it offers, in addition to the standard DBT treatment, an intensive DBT program, the duration of which is reduced to 1 month, with daily group and individual sessions (Perroud et al. 2010b).

The patients are referred to us by private psychiatrists or by the psychiatric hospital. The population that undergoes our BPD program is particular as it is nearly exclusively made up of women. Women represent the large majority of those who contact us for a clinical assessment. Furthermore, among the male patients referred to us, some show criteria that exclude them from the program, such as substance addiction and legal problems.

The study was approved by the ethics committee of the University Hospitals of Geneva (HUG), and a written informed consent form was signed by all participants.

\section{Participants}

For the purpose of this study, we recruited 166 patients who suffer from BPD and who are being treated in our therapy program. In this group of 166 BPD sufferers, 67 also had adult ADHD as a comorbidity. Among these 67 
patients, 29 underwent a treatment with MPH- or desmethylphenidate (dMPH)-based stimulants, whereas the 38 others did not receive the treatment. As we have already mentioned, the diagnosis of adult ADHD is established upon admission and based on a clinical interview conducted by an experienced psychiatrist. The decision to treat the patient with stimulants was made when ADHD was deemed to be hampering the capacity of the subjects to follow the therapy. Thus, not all ADHD comorbid patients were treated. Treatment was started just before the beginning of DBT treatment with an equivalent of $10 \mathrm{mg}$ of MPH, later adjusted up to an equivalent of $80 \mathrm{mg}$ according to individual responses (Faraone and Glatt 2010; Volkow and Swanson 2013). None of the participants who were offered MPH treatment declined to take it. Treatment was stopped if patients complained of unbearable adverse side effects or when instances of non-response were observed. Worthy of note is the fact that non-responders to MPH or those who had to stop this medication due to side effects $(N=8)$ were removed from subsequent analyses (see Fig. 1). Other treatments at admission usually remained unchanged but some may have been adjusted according to clinical status. For instance, if a participant suffered from a severe depressive episode without any improvement after few weeks, his/her treatment was adjusted accordingly (increased of dosage, augmentation strategies, or change of molecule). Furthermore, only eight subjects (all BPD without ADHD comorbidity) saw a change in their other medications after entering our program.

\section{Measurements}

Before being admitted in our therapy program, patients undergo a clinical assessment interview conducted by two members of the treatment team, one of whom is either an experienced psychiatrist or an experienced psychologist. The purpose of the interview is to confirm the presence of $\mathrm{BPD}$, of ADHD, and to exclude any medical condition or axis-I disorder that might better explain the clinical profile of the patient. Patients then undergo two semi-structured interviews conducted by specially trained psychologists. The first is a Diagnostic Interview for Genetic Studies (DIGS), which systematically assesses axis-I comorbidities along with ADHD symptoms during childhood and their persistence into adulthood according to DSM-IV criteria (Preisig et al. 1999). The DIGS uses 13 questions for attentional symptoms and 10 questions for hyperactiveimpulsive symptoms, in order to capture the full spectrum of the disorder. For the severity of ADHD, the mean value obtained by the sum of these questions was used (see Table 1). The second is a structured clinical interview

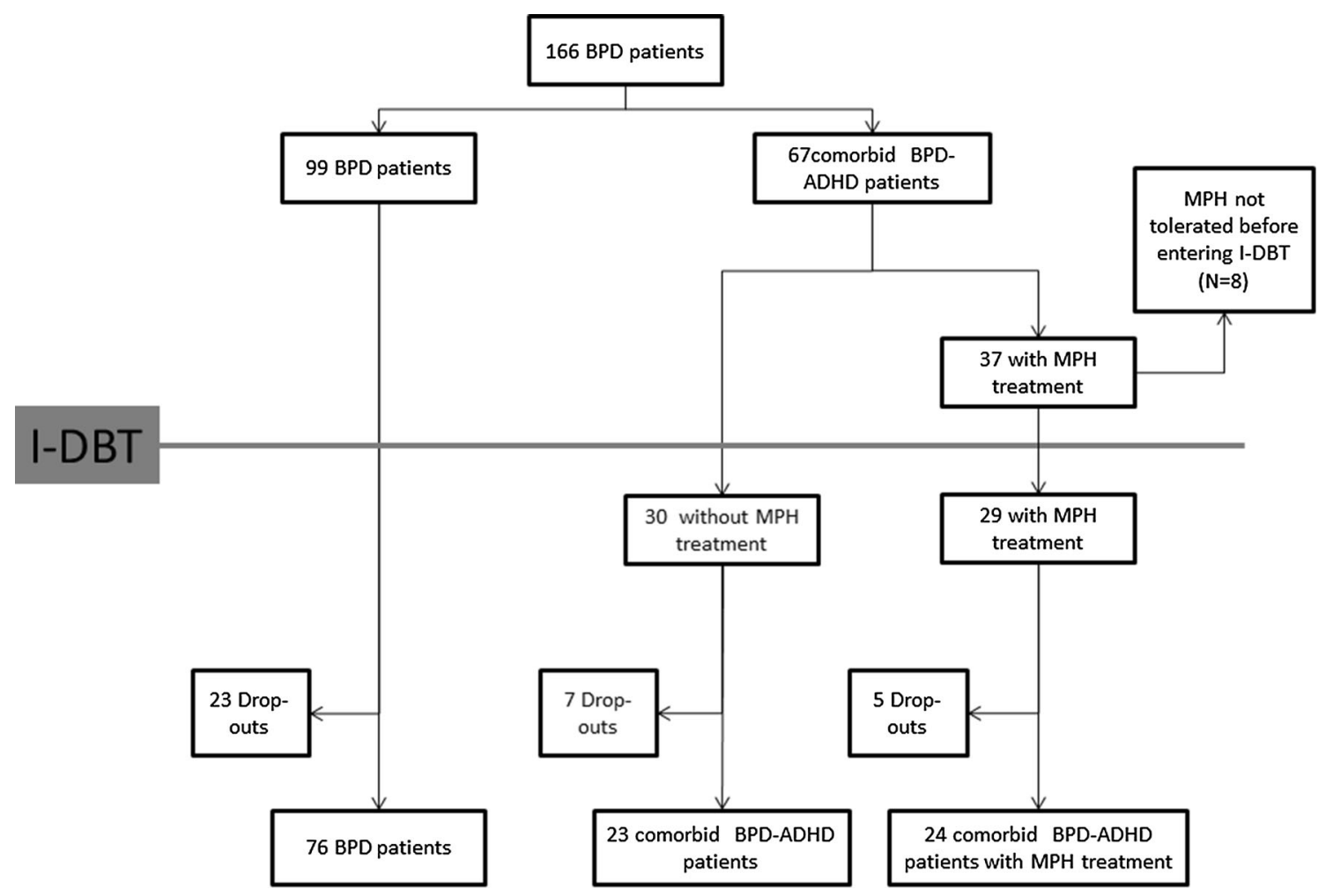

Fig. 1 Flow chart of participants throughout the study. Decision to treat with MPH was made when ADHD was considered by the clinician to be enough severe to hamper the capacity of the subjects to follow the therapy (I-DBT) 
Table 1 Clinical and demographic characteristics of the three clinical groups at baseline

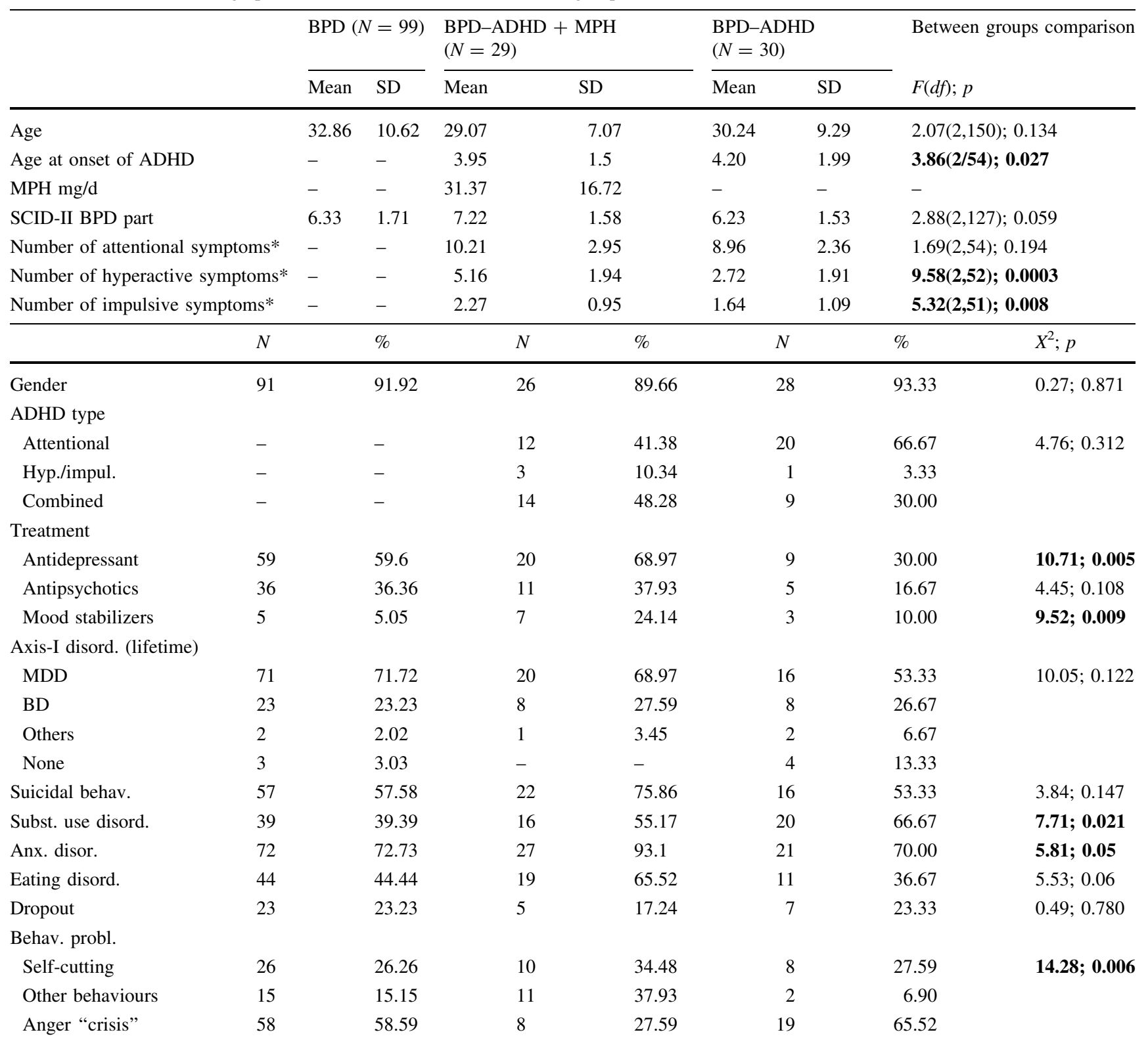

BPD patients (BPD), BPD patients with comorbid ADHD receiving MPH-based treatment (BPD-ADHD + MPH), and BPD patients with comorbid ADHD not receiving MPH-based treatment (BPD-ADHD). Significant results are bolded

* Numbers based on the DIGS: 13 questions for the attentional symptoms, 7 for the hyperactive symptoms, and 3 for the impulsive symptoms

conducted to diagnose BPD (SCID-II BPD part) (First et al. 1994). Only patients who satisfy at least five of the nine criteria for SCID-II BPD are considered for the purposes hereof. With regard to ADHD, the presence of the disorder before the age of seven (according to DSM-IV) is required, based on the DIGS and the Wender Utah Rating Scale (WURS), which includes 25 questions rated on a Likert five-point scale, and for which we used the particularly high threshold of 46 to establish a diagnosis of childhood ADHD (Ward et al. 1993; Fossati et al. 2002).
If the first interview seems to indicate the presence of ADHD-compatible symptoms, the patient undergoes a clinical interview with a senior psychiatrist at our centre, focusing on adult ADHD.

In addition to these diagnoses and semi-structured interviews, patients are required to complete the following forms upon being admitted to the program and again after 1 month of specialised treatment: the adult ADHD SelfReport Scale (ASRS v.1.1) to assess the severity of adult ADHD (Romo et al. 2010), the Barratt Impulsiveness Scale 
(BIS-10) that measures three dimensions of impulsiveness (motor and behaviour, cognitive and attentional, and nonplanning) (Bayle et al. 2000), the State-Trait Anger Expression (STAXI) that measures expressions and experiences of anger (Spielberger 1998), the Beck Depression Inventory II (BDI-II) to assess the severity and symptoms of depression (Beck et al. 1996), and finally the Beck Hopelessness Scale (BHS) that assesses the degree of pessimism regarding the future (BHS; Beck et al. 1974).

Other information, such as dysfunctional behaviours, was retrieved from the medical charts and from the contract signed by the patients when entering DBT, where targeted dysfunctional behaviours are listed.

All the patients of our centre are invited to take part in our clinical research activities. Those who decline receive the same treatment and undergo the same clinical and approval interviews as the other patients, but their data are not retrieved from their medical file.

\section{Statistical analyses}

ANOVA and Chi-square tests were used to compare baseline characteristics between groups. Mixed linear models with hierarchical random effects of individual fitted with maximum likelihood were used to assess response to treatment in the entire sample (Perroud et al. 2010b). The subject-specific random intercept was included in order to relax the assumption of conditional independence in the responses of the same person, thereby minimising type I errors. Potential predictors of treatment response were included as fixed factors. Values were standardised and may thus be interpreted as effect sizes. ANOVA with percentage of changes for each variable of interest as dependant variables was used to compare response to treatment between the three different clinical groups (BPD, BPD-ADHD treated, and BPD-ADHD untreated). As other treatments (antidepressants and mood stabilizers) differed among groups, other treatments were used as covariate in the models (Table 1). A $p$ value $<0.05$ was used as a threshold for significance. All analyses were done using STATA v12.

\section{Results}

Table 1 displays the patients' clinical and demographic characteristics. Comorbid BPD-ADHD patients undergoing MPH treatment differed from comorbid BPD-ADHD patients who forewent such treatment in terms of age at onset of $\operatorname{ADHD}\left(F_{(6,54)}=3.86 ; p=0.03\right)$ and number of hyperactive and/or impulsive symptoms $\left(F_{(2,52)}=9.58\right.$; $p=0.0003$ and $\left.F_{(2,51)}=5.32 ; p=0.008\right)$. Comorbid BPD-ADHD patients undergoing MPH treatment showed a trend towards higher SCID-II BPD part scores than the two other groups $\left(F_{(2,127}=2.88 ; p=0.059\right)$. This was also reflected by more severe dysfunctional behaviours than the other groups (more self-cuttings and fewer anger crises and other behaviours) $\left(X^{2}=14.28 ; p=0.006\right)$. As mentioned above, psychopharmacological treatments (antidepressants and mood stabilizers) differed among groups (Table 1).

At baseline, comorbid BPD-ADHD patients undergoing or foregoing MPH treatment showed a degree of impulsiveness that was significantly higher than that of BPD patients $\left(F_{(2,138)}=10.61 ; p=0.0001\right)$ for BIS-10 total score. This was mainly explained by increased motor impulsiveness (11 items, e.g. "I act on impulse" or "I buy things on impulse") $\left(F_{(2,140)}=15.60 ; p<0.0001\right)$. The three groups also differed at baseline in terms of ASRS v.1.1 total score and depression severity $\left(F_{(2,140)}=14.49\right.$; $p<0.0001$ and $\left.F_{(2,142)}=5.32 ; 0.006\right)$ (Table 2).

\section{Retention of participants}

Thirty-five subjects dropped out during the I-DBT $(22.2 \%)$. There was no difference between groups in dropout rates $\left(X^{2}=0.49 ; p=0.780\right)$ (Table 1$)$. A history of suicide attempts was associated with a lower dropout rate; only $32.14 \%(N=9)$ of those who dropped out reported having attempted suicide, whereas more than $75 \%$ $(N=86)$ of those remaining in the therapy had attempted suicide in the past $\left(X^{2}=18.35 ; p<0.0001\right)$. This was also reflected by fewer self-cutting behaviours among participants who dropped out, compared to those who remained in the study (Table 3). Antidepressant administration further reduced dropout rates; $61.8 \%(N=76)$ of those who remained in the therapy were administered an antidepressant, whereas this was the case only for $34.3 \%(N=12)$ of those who dropped out $\left(X^{2}=8.35 ; p=0.004\right)$. A comorbid anxiety disorder was also associated with a lower dropout rate. Finally, increased dosage in the MPH treatment reduced the participants' dropout rate (Table 3).

\section{Response to intensive DBT}

In the whole sample, with the exception of BIS-10 motor impulsiveness, there was a significant decrease in all dimensions ranging from small (for BIS-10 total score, for example), medium (for State Anger, Anger Out, and hopelessness, for instance), to large effect sizes (for depression severity) (Table 4) during the 4-week intensive DBT. Among the variables that distinguish the three groups at baseline, displaying self-cutting behaviours as opposed to anger crisis and/or other behaviours was associated with an improved response to I-DBT $\left(F_{(2,106)}=3.27 ; p=0.04\right.$ when considering response in terms of reduction in depression severity). No other significant baseline characteristics between the three groups predicted outcome. 
Table 2 Comparison of the evolution of the scores between BPD patients (BPD), BPD patients with comorbid ADHD receiving MPH-based treatment (BPD-ADHD + MPH), and BPD patients with comorbid ADHD not receiving MPH-based treatment (BPD-ADHD)

\begin{tabular}{|c|c|c|c|c|c|c|c|c|c|c|c|c|c|}
\hline & \multicolumn{4}{|c|}{ A. $\operatorname{BPD}(N=76)^{*}$} & \multicolumn{4}{|c|}{$\begin{array}{l}\text { B. BPD-ADHD + MPH } \\
(N=24)^{*}\end{array}$} & \multicolumn{4}{|c|}{ C. BPD-ADHD $(N=23)^{*}$} & \multirow{3}{*}{$\begin{array}{l}\text { Between groups } \\
\text { comparison }\end{array}$} \\
\hline & \multicolumn{2}{|c|}{ Baseline } & \multicolumn{2}{|c|}{ Post-treatment } & \multicolumn{2}{|c|}{ Baseline } & \multicolumn{2}{|c|}{ Post-treatment } & \multicolumn{2}{|c|}{ Baseline } & \multicolumn{2}{|c|}{ Post-treatment } & \\
\hline & Mean & SD & Mean & SD & Mean & SD & Mean & SD & Mean & SD & Mean & SD & \\
\hline \multicolumn{14}{|l|}{ BIS-10 } \\
\hline Total score & 64.06 & 16.72 & 62.30 & 14.60 & 78.37 & 19.54 & 70.10 & 21.59 & 77.35 & 14.04 & 76.11 & 15.20 & $3.31_{(2,94)} ; 0.04$ \\
\hline Motor & 21.12 & 7.05 & 20.67 & 7.06 & 29.47 & 7.95 & 25.70 & 9.60 & 25.91 & 6.64 & 26.77 & 7.14 & $3.61_{(2,97)} ; 0.03$ \\
\hline Attentional & 22.03 & 6.75 & 21.24 & 5.58 & 25.14 & 5.91 & 23.07 & 8.36 & 25.86 & 5.83 & 24.83 & 6.53 & $1.08_{(2,97)} ; 0.34$ \\
\hline Non-planning & 21.17 & 7.06 & 20.39 & 6.47 & 23.75 & 9.26 & 20.84 & 8.59 & 25.57 & 6.54 & 24.50 & 5.88 & $0.34_{(2,94)} ; 0.71$ \\
\hline \multicolumn{14}{|l|}{ STAXI } \\
\hline Trait Anger & 27.45 & 6.77 & 24.97 & 6.62 & 29.30 & 7.34 & 24.61 & 7.01 & 26.48 & 6.26 & 26.05 & 7.92 & $3.11_{(2,98)} ; 0.05$ \\
\hline State Anger & 23.60 & 8.77 & 18.48 & 6.28 & 26.44 & 8.35 & 17.52 & 5.71 & 22.25 & 8.34 & 18.33 & 7.49 & $3.19_{(2,98)} ; 0.04$ \\
\hline Anger In & 21.54 & 4.62 & 21.05 & 5.52 & 23.74 & 5.56 & 21.78 & 5.54 & 20.94 & 5.94 & 19.65 & 6.69 & $0.71_{(2,98)} ; 0.49$ \\
\hline Anger Out & 18.67 & 5.54 & 16.35 & 4.54 & 21.28 & 7.05 & 17.01 & 5.89 & 18.63 & 4.22 & 16.69 & 4.98 & $0.07_{(2,98)} ; 0.93$ \\
\hline Anger control & 18.81 & 4.77 & 19.93 & 4.08 & 18.41 & 4.66 & 20.04 & 4.78 & 19.34 & 5.10 & 20.27 & 4.75 & $0.47_{(2,98)} ; 0.62$ \\
\hline ASRS v1.1 total score & 38.10 & 10.71 & 36.53 & 9.39 & 51.07 & 12.97 & 45.32 & 12.29 & 41.34 & 10.18 & 41.50 & 11.02 & $4.29_{(2,93)} ; 0.02$ \\
\hline BDI & 34.03 & 11.68 & 21.09 & 13.04 & 40.46 & 9.52 & 19.26 & 10.96 & 31.61 & 14.24 & 23.83 & 16.16 & $4.03_{(2,99)} ; 0.02$ \\
\hline Hopelessness & 11.11 & 4.44 & 8.23 & 4.92 & 12.54 & 5.58 & 8.54 & 5.94 & 11.24 & 5.43 & 11.01 & 5.28 & $0.02_{(2,100)} ; 0.97$ \\
\hline
\end{tabular}

Significant differences are bolded. Analyses (ANOVA) were adjusted on the following medications: antidepressants, antipsychotics, mood stabilizers

* Number of subjects who completed the study

There was a significant difference in response to treatment between the three groups with regard to motor impulsiveness $\left(F_{(2,97)}=3.61 ; p=0.03\right)$ and BIS-10 total score $\left(F_{(2,94)}=3.31 ; p=0.04\right)$, Trait and State Anger scores $\quad\left(F_{(2,98)}=3.11 ; \quad p=0.05 \quad\right.$ and $\quad F_{(2,98)}=3.19$; $p=0.04)$, ADHD severity $\left(F_{(2,93)}=4.29 ; p=0.02\right)$, and depression severity $\left(F_{(2,99)}=4.03 ; p=0.02\right)$. More specifically, compared to BPD-ADHD without stimulant, BPD-ADHD undergoing MPH treatment showed a significantly improved response to DBT treatment with regard to BIS-10 motor impulsiveness (mean value of individuals' improvement percentage: 4.96 vs. $15.87 \%$, respectively; $F_{(1 / 38)}=4.38 ; p=0.043$ ), BIS-10 total score (mean value of individuals' improvement percentage: 8.43 vs. $14.66 \%$, respectively; $\left.F_{(1 / 38)}=3.98 ; p=0.05\right)$, State Anger score (mean value of individuals' improvement percentage: 16.68 vs. $28.92 \%$, respectively; $F_{(1 / 38)}=6.89 ; p=0.01$ ), depression severity $\left(29.95\right.$ vs. $54.25 \% ; F_{(1 / 38)}=9.53$; $p=0.004)$, and ADHD severity (3.90 vs. $13.11 \%$; $\left.F_{(1 / 38)}=6.20 ; p=0.017\right)$ (Table 2).

\section{Discussion}

The main finding of this study is that treating comorbid BPD-ADHD subjects with MPH, as opposed to not treating them, was associated with greater improvements in several dimensions, which include, unsurprisingly, ADHD severity and impulsiveness but also, and this is the more striking observation, depression severity and the tendency to express anger. Indeed, the changes in ASRS v1.1 scores before and after the treatment, although of moderate magnitude, showed a clearly favourable evolution of values for MPH-treated comorbid BPD-ADHD patients compared to non-treated comorbid patients. Similarly, Trait-State Anger and BDI scores, reflecting lifetime and current expression of anger and depression severity, were more significantly reduced among MPH-treated patients compared to nonMPH-treated ones. A similar trend was observed for motor impulsiveness and BIS-10 total scores. It thus appears that the intensive psychotherapeutic approach afforded to comorbid BPD-ADHD patients is more efficient when used with MPH medication. A MPH-based treatment can significantly improve the clinical course, on the one hand because of its effect on ADHD symptoms, and on the other by improving the availability of the patient to therapy. This last hypothesis remains to be verified. Similar to these findings, studies showed improvement in BPD symptoms in patients treated with bupropion and duloxetine (Bellino et al. 2010; Perroud et al. 2010a). Those two treatments also inhibit dopamine and norepinephrine reuptake, respectively, which might explain their efficacy. In this 
Table 3 Comparison of participants who dropped out with participants who remained in the study
Significant differences are bolded and italicised

\begin{tabular}{|c|c|c|c|c|c|c|c|}
\hline & & \multicolumn{2}{|c|}{ Dropouts $(N=35)$} & \multicolumn{2}{|c|}{ Non-dropouts $(N=123)$} & \multirow[t]{2}{*}{$t$} & \multirow[t]{2}{*}{$p$} \\
\hline & & Mean & SD & Mean & SD & & \\
\hline \multicolumn{2}{|l|}{ Age } & 30.43 & 10.09 & 32.02 & 9.91 & -0.45 & 0.64 \\
\hline \multicolumn{2}{|c|}{ Age at onset of ADHD } & 4.17 & 1.27 & 4.51 & 2.62 & -0.43 & 0.66 \\
\hline \multicolumn{2}{|l|}{ MPH mg/d } & 14.00 & 8.94 & 35.00 & 15.72 & -2.86 & 0.008 \\
\hline \multicolumn{2}{|l|}{ SCID-II BPD part } & 6.21 & 1.87 & 6.52 & 1.74 & -0.82 & 0.41 \\
\hline \multicolumn{2}{|c|}{ Number of attentional symptoms* } & 9.58 & 2.64 & 9.08 & 2.88 & 0.39 & 0.69 \\
\hline \multicolumn{2}{|c|}{ Number of hyperactive symptoms* } & 3.33 & 1.92 & 3.49 & 2.28 & -0.37 & 0.71 \\
\hline \multicolumn{2}{|c|}{ Number of impulsive symptoms* } & 1.75 & 0.97 & 1.69 & 1.19 & -0.03 & 0.97 \\
\hline \multicolumn{8}{|l|}{ BIS-10 } \\
\hline \multicolumn{2}{|l|}{ Total score } & 68.13 & 18.95 & 69.61 & 17.45 & -0.37 & 0.71 \\
\hline \multicolumn{2}{|l|}{ Motor } & 23.05 & 8.14 & 23.88 & 7.80 & -0.44 & 0.66 \\
\hline \multicolumn{2}{|l|}{ Attentional } & 23.09 & 7.57 & 23.49 & 6.25 & -0.28 & 0.78 \\
\hline \multicolumn{2}{|l|}{ Non-planning } & 22.00 & 7.28 & 22.36 & 7.66 & -0.27 & 0.79 \\
\hline \multicolumn{8}{|l|}{ STAXI } \\
\hline \multicolumn{2}{|l|}{ Trait Anger } & 26.73 & 6.79 & 27.75 & 6.69 & -0.74 & 0.46 \\
\hline \multicolumn{2}{|l|}{ State Anger } & 23.86 & 7.95 & 23.83 & 8.58 & 0.01 & 0.98 \\
\hline \multicolumn{2}{|l|}{ Anger In } & 21.11 & 5.41 & 21.79 & 5.24 & -0.62 & 0.53 \\
\hline \multicolumn{2}{|l|}{ Anger Out } & 17.61 & 5.29 & 19.63 & 5.70 & -1.74 & 0.08 \\
\hline \multicolumn{2}{|l|}{ Anger control } & 20.00 & 5.14 & 18.48 & 4.59 & 1.57 & 0.11 \\
\hline ASRS v1.1 total sco & & 40.34 & 12.70 & 41.47 & 11.56 & -0.46 & 0.64 \\
\hline BDI & & 31.14 & 12.84 & 34.69 & 11.98 & -1.41 & 0.16 \\
\hline Hopelessness & & 9.97 & 5.10 & 11.53 & 4.73 & -1.58 & 0.12 \\
\hline & $N$ & $\%$ & $N$ & $\%$ & $X^{2}$ & 1 & $p$ \\
\hline Gender & 30 & 85.71 & 115 & 93.50 & 2.18 & & 0.139 \\
\hline ADHD type & & & & & & & \\
\hline Attentional & 6 & 50.00 & 26 & 55.32 & 1.54 & & 0.46 \\
\hline Hyp./impul. & 0 & 0.00 & 4 & 8.51 & & & \\
\hline Combined & 6 & 50.00 & 17 & 36.17 & & & \\
\hline Treatment & & & & & & & \\
\hline Antidepressant & 12 & 34.29 & 76 & 61.79 & 8.35 & & 0.004 \\
\hline Antipsychotics & 8 & 22.86 & 44 & 35.77 & 2.06 & & 0.151 \\
\hline Mood stabilizers & 1 & 2.86 & 14 & 11.38 & 2.3 & & 0.129 \\
\hline Axis-I disord. (lifeti & & & & & & & \\
\hline MDD & 23 & 65.71 & 84 & 68.29 & 3.09 & & 0.378 \\
\hline $\mathrm{BD}$ & 7 & 20.00 & 32 & 26.02 & & & \\
\hline Others & 2 & 5.71 & 3 & 2.44 & & & \\
\hline None & 3 & 8.57 & 4 & 3.25 & & & \\
\hline Suicidal behav. & 9 & 32.14 & 86 & 74.78 & 18.35 & & $<0.0001$ \\
\hline Subst. use disord. & 16 & 45.71 & 59 & 47.97 & 0.05 & & 0.81 \\
\hline Anx. disor. & 22 & 62.86 & 98 & 79.67 & 4.21 & & 0.04 \\
\hline Eating disord. & 13 & 37.14 & 61 & 49.59 & 1.69 & & 0.19 \\
\hline Behav. probl. & & & & & & & \\
\hline Self-cutting & 5 & 14.29 & 39 & 31.97 & 7.44 & & 0.024 \\
\hline Other behaviours & 4 & 11.43 & 24 & 19.67 & & & \\
\hline Anger "crisis" & 26 & 74.29 & 59 & 48.34 & & & \\
\hline
\end{tabular}

whatever the nature of their comorbidity; this, for instance, has already been suggested for ADHD patients suffering from bipolar disorder (Perroud et al. 2014). 
Table 4 Evolution of BIS-10, STAXI, ASRS v1.1, BDI, and BHS during I-DBT in the entire sample

Significant results are bolded. Analyses were done using mixed linear models, and thus, all subjects $(N=158)$ were used in the analyses

\begin{tabular}{|c|c|c|c|c|c|c|}
\hline & \multicolumn{4}{|c|}{$\operatorname{BPD}(N=158)$} & \multirow{3}{*}{$b$} & \multirow{3}{*}{$p$} \\
\hline & \multicolumn{2}{|c|}{ Baseline } & \multicolumn{2}{|c|}{ Post-treatment } & & \\
\hline & Mean & SD & Mean & SD & & \\
\hline \multicolumn{7}{|l|}{ BIS-10 } \\
\hline Total score & 69.15 & 18.12 & 66.19 & 17.06 & -0.15 & 0.005 \\
\hline Motor & 23.55 & 7.89 & 22.74 & 8.06 & -0.09 & 0.214 \\
\hline Attentional & 23.28 & 6.62 & 22.22 & 6.50 & -0.14 & $\mathbf{0 . 0 3 3}$ \\
\hline Non-planning & 22.43 & 7.61 & 21.17 & 6.96 & -0.18 & 0.005 \\
\hline \multicolumn{7}{|l|}{ STAXI } \\
\hline Trait Anger & 27.65 & 6.81 & 25.07 & 6.88 & -0.38 & $<0.0001$ \\
\hline State Anger & 23.94 & 8.66 & 18.25 & 6.33 & -0.75 & $<0.0001$ \\
\hline Anger In & 21.87 & 5.10 & 20.97 & 5.77 & -0.19 & 0.035 \\
\hline Anger Out & 19.17 & 5.73 & 16.55 & 4.88 & -0.53 & $<0.0001$ \\
\hline Anger control & 18.82 & 4.78 & 20.01 & 4.31 & 0.26 & 0.0002 \\
\hline ASRS v1.1 total score & 41.18 & 12.12 & 39.25 & 10.87 & -0.16 & 0.006 \\
\hline BDI & 34.87 & 12.03 & 21.16 & 13.16 & -0.98 & $<0.0001$ \\
\hline Hopelessness & 11.40 & 4.84 & 11.35 & 4.83 & -0.64 & $<0.0001$ \\
\hline
\end{tabular}

These results underline the importance of detecting ADHD as a comorbidity among patients treated for BPD, not only because it helps to better reduce ADHD symptoms, such as motor impulsiveness, but also because it is associated with better effects on symptoms more closely related to BPD, such as anger management. For instance, MPH treatment was associated with significant reduction in depression severity in our study. Though our results remain controversial, they are in line with previous findings, suggesting that MPH augmentation of antidepressants and more generally of treatments with psychostimulants might be associated with moderate or marked improvement in depression, or at least improvement in some domains related to depression, such as fatigue and apathy (Mendonca et al. 2007; Ravindran et al. 2008; McIntyre et al. 2013; Trivedi et al. 2013; Sinita and Coghill 2014). Nevertheless, our findings concerning comorbid BPD-ADHD need further investigation before any conclusion can be drawn as to the role of MPH in helping to treat depression in this population.

The improvement of depressive symptoms in comorbid BPD-ADHD subjects might also be the consequence of the more effective antidepressant treatment provided to them, as our results show. Some of these antidepressants, mainly those acting through the inhibition of dopamine reuptake, such as bupropion, might also participate in the reduction in ADHD severity among these subjects (Maneeton et al. 2014). Indeed, with regard to other medication, one of the differences between MPH-treated and MPH-untreated comorbid BPD-ADHD patients resides in the antidepressant treatment followed by patients upon admission. BPDADHD comorbid patients undergoing MPH treatment were, upon admission, more frequently treated with antidepressants than those who did not receive the stimulantbased treatment. The fact that BPD-ADHD comorbid patients undergoing MPH treatment had higher depression scores at their admission in our specialised program on the one hand, and the fact that they were referred to us as suffering from BPD but not diagnosed as ADHD subjects on the other suggest that antidepressants were given to these patients in order to treat the poor clinical presentation of these subjects, a poor clinical presentation possibly better explained by the presence of ADHD than of any depressive spectrum disorders. In reality, MPH-untreated comorbid BPD-ADHD patients did not receive as much antidepressant treatment as non-comorbid BPD patients, a fact that does not contradict the idea that the baseline BDI was less severe among the former group. All these findings suggest that ADHD in BPD is associated with a more depressive clinical presentation and is therefore not be responsive to classical antidepressants, but to MPH.

We also found that a higher degree of severity of ADHD symptoms was associated with a greater likelihood of receiving treatment. Indeed, when the two BPD-ADHD groups were compared in terms of the ADHD dimension, it appeared that the treated group showed increased severity, as evidenced by the increase in hyperactivity/impulsive symptoms. This raises the question of the existence of a relation between observed increased severity and the clinical decision to treat those patients with a stimulant-based treatment. This seemingly trivial observation may be of interest, as it suggests that specialists trained in the recognition of these two closely related disorders are able to distinguish symptoms more related to BPD from those 
more related to ADHD, which may not necessarily be the case in other clinical settings (Perroud et al. 2014).

We also observed a history of increased self-harming behaviours among BPD-ADHD patients undergoing treatment compared with the group not receiving treatment. It appears, therefore, that the level of severity of this subgroup in terms of impulsiveness, which was in part reflected by higher SCID-II BPD part scores (which include items directly related to impulsiveness), and in terms of action taking, is possibly greater. This may suggest that ADHD may increase the severity of BPD.

Overall and as previously demonstrated, intensive DBT was effective in reducing most of the dimensions related to BPD, but also those related to ADHD. In our original investigations (Perroud et al. 2010b, 2012), we found that intensive DBT significantly reduced depression, hopelessness, and increased mindfulness skills. The current findings add to these preliminary results, showing that this psychotherapeutic approach is also associated with improved impulsiveness and anger management, two dimensions more closely related to BPD.

This study has several limitations, the first of which being that both groups of comorbid patients appear to show different levels of severity in terms of baseline symptoms. As BPD-ADHD patients receiving MPH treatment showed greater levels of depression and ADHD symptoms to begin with, our results are maybe better explained by a regression to the mean. Despite this potentially confounding factor, we, as clinicians who have witnessed the great improvement of patients taking MPH, strongly believe that the current findings reflect true clinical changes that these subjects undergo over time. Nevertheless, the non-blinded design of our study might have influenced the results, insofar as clinicians might have expected a better treatment response among those treated by MPH than among those not receiving such medication. However, the fact that participants were reporting their own impressions and symptoms in self-report questionnaires provides partial protection against this bias. Patients were not blinded to the intervention (MPH treatment), and this might also have biased our results. However, after entering DBT, no further attention was given to MPH treatment (no more than antidepressants for instance), and no modification in the follow-up in relation to this particular medication was done. Then, and as highlighted in our previous articles, there is the absence of a control group that is not subject to intensive DBT. Thirdly, the clinical population is nearly exclusively made of women, as the male population of BPD and ADHD disorders is hardly examined here. Another limitation to the generalizability of our findings is that the studied patients were those who signed up for an intensive course of DBT. This group might not be representative of all BPD patients. For instance, one prerequisite of our program is that patients should have an occupation, even voluntary. This precludes any generalisability to patients without any occupational activities.

A further limitation is the outpatient nature of the healthcare centre, which, along with high admission numbers, renders access to highly impulsive patients difficult. Finally, we did not apply a correction for multiple testing, and our results should thus be taken with caution, as type 1 errors might occur for some of them. As discussed above for the regression to the mean, and as clinicians, we strongly believe that our findings reflect true clinical changes that affect these subjects over time.

Twenty-two percentage of participants dropped out during the 4-week I-DBT course, a rate slightly higher but still within the range of our previous findings (ref). Although no significant difference was found in terms of dropout rates between groups, the dropout rate $(17 \%)$ among BPD-ADHD patients receiving MPH treatment was still lower than in the two other groups ( $23 \%)$, thus reinforcing previous observations that DBT is very well suited to patients suffering from ADHD, but even more so to those receiving MPH medication. In addition, a higher dosage of MPH was associated with lower dropout rates, suggesting that proper titration of this medication might not only improve response to treatment but also prevents sudden withdrawal from the psychotherapeutic setting.

\section{Conclusions}

This naturalistic study highlights the importance of systematically screening BPD patients for comorbidity. The treatment of these comorbidities is part of the personality disorder therapy and can, when adequately provided, greatly contribute to clinical improvement. Therefore, a MPH-based treatment will improve the symptoms of comorbid BPD-ADHD patients who display high impulsiveness scores.

Acknowledgments We would like to thank Gérald Bouillault, JeanJacques Kunckler, Sophie Blin, Charlotte Germond, Venus Kaby, Karen Dieben and Caroline Waeber for their help in recruiting and assessing patients.

Conflict of interest Authors have no personal affiliations or financial relationships with any commercial interest to disclose in relation to this article.

\section{References}

American Psychiatric Association (2001) Practice guideline for the treatment of patients with borderline personality disorder. American Psychiatric Association. Am J Psychiatry 158(10 Suppl): $1-52$ 
Bateman A, Fonagy P (2010) Mentalization based treatment for borderline personality disorder. World Psychiatry 9(1):11-15

Bayle FJ, Bourdel MC, Caci H et al (2000) Factor analysis of french translation of the Barratt impulsivity scale (BIS-10). Can J Psychiatry 45(2): 156-165

Beck AT, Weissman A, Lester D et al (1974) The measurement of pessimism: the hopelessness scale. J Consult Clin Psychol 42(6):861-865

Beck AT, Steer RA, Ball R et al (1996) Comparison of beck depression inventories-IA and -II in psychiatric outpatients. J Pers Assess 67(3):588-597

Bellino S, Paradiso E, Bozzatello P et al (2010) Efficacy and tolerability of duloxetine in the treatment of patients with borderline personality disorder: a pilot study. J Psychopharmacol 24(3):333-339

Davids E, Gastpar M (2005) Attention deficit hyperactivity disorder and borderline personality disorder. Prog Neuropsychopharmacol Biol Psychiatry 29(6):865-877

Faraone SV, Glatt SJ (2010) A comparison of the efficacy of medications for adult attention-deficit/hyperactivity disorder using meta-analysis of effect sizes. J Clin Psychiatry 71(6):754-763

First M, Gibbon M, Spitzer R et al (1994) Structured Clinical Interview for DSM-IV personality disorders (SCID-II). American Psychiatric Association, Washington, DC

Fossati A, Novella L, Donati D et al (2002) History of childhood attention deficit/hyperactivity disorder symptoms and borderline personality disorder: a controlled study. Compr Psychiatry 43(5):369-377

Golubchik P, Sever J, Zalsman G et al (2008) Methylphenidate in the treatment of female adolescents with cooccurrence of attention deficit/hyperactivity disorder and borderline personality disorder: a preliminary open-label trial. Int Clin Psychopharmacol 23(4):228-231

Herpertz S, Gretzer A, Steinmeyer EM et al (1997) Affective instability and impulsivity in personality disorder. Results of an experimental study. J Affect Disord 44(1):31-37

Herpertz SC, Kunert HJ, Schwenger UB et al (1999) Affective responsiveness in borderline personality disorder: a psychophysiological approach. Am J Psychiatry 156(10):1550-1556

Ingenhoven T, Lafay P, Rinne $\mathrm{T}$ et al (2010) Effectiveness of pharmacotherapy for severe personality disorders: meta-analyses of randomized controlled trials. J Clin Psychiatry 71(1):14-25

Jacob GA, Gutz L, Bader K et al (2010) Impulsivity in borderline personality disorder: impairment in self-report measures, but not behavioral inhibition. Psychopathology 43(3):180-188

Lampe K, Konrad K, Kroener S et al (2007) Neuropsychological and behavioural disinhibition in adult ADHD compared to borderline personality disorder. Psychol Med 37(12):1717-1729

Lieb K, Zanarini MC, Schmahl C et al (2004) Borderline personality disorder. Lancet 364(9432):453-461

Linehan MM (1993) Cognitive-behavioral treatment of borderline personality disorder. The Guilford Press, New York

Lynch TR, Trost WT, Salsman N et al (2007) Dialectical behavior therapy for borderline personality disorder. Annu Rev Clin Psychol 3:181-205

Maneeton N, Maneeton B, Intaprasert S et al (2014) A systematic review of randomized controlled trials of bupropion versus methylphenidate in the treatment of attention-deficit/hyperactivity disorder. Neuropsychiatr Dis Treat 10:1439-1449

Matthies S, Philipsen A, Lackner HK et al (2014) Regulation of sadness via acceptance or suppression in adult attention deficit hyperactivity disorder (ADHD). Psychiatry Res 220(1-2):461-467

McIntyre RS, Alsuwaidan M, Soczynska JK et al (2013) The effect of lisdexamfetamine dimesylate on body weight, metabolic parameters, and attention deficit hyperactivity disorder symptomatology in adults with bipolar I/II disorder. Hum Psychopharmacol 28(5):421-427

Mendonca DA, Menezes K, Jog MS (2007) Methylphenidate improves fatigue scores in Parkinson disease: a randomized controlled trial. Mov Disord 22(14):2070-2076

Nigg JT (2001) Is ADHD a disinhibitory disorder? Psychol Bull 127(5):571-598

Perroud N, Salzmann A, Saiz PA et al (2010a) Rare genotype combination of the serotonin transporter gene associated with treatment response in severe personality disorder. Am J Med Genet B Neuropsychiatr Genet 153B(8):1494-1497

Perroud N, Uher R, Dieben K et al (2010b) Predictors of response and drop-out during intensive dialectical behavior therapy. J Pers Disord 24(5):634-650

Perroud N, Nicastro R, Jermann F et al (2012) Mindfulness skills in borderline personality disorder patients during dialectical behavior therapy: preliminary results. Int $\mathrm{J}$ Psychiatry Clin Pract 16(3): 189-196

Perroud N, Cordera P, Zimmermann J et al (2014) Comorbidity between attention deficit hyperactivity disorder (ADHD) and bipolar disorder in a specialized mood disorders outpatient clinic. J Affect Disord 168:161-166

Philipsen A (2006) Differential diagnosis and comorbidity of attention-deficit/hyperactivity disorder (ADHD) and borderline personality disorder (BPD) in adults. Eur Arch Psychiatry Clin Neurosci 256(Suppl 1):i42-i46

Philipsen A, Limberger MF, Lieb K et al (2008) Attention-deficit hyperactivity disorder as a potentially aggravating factor in borderline personality disorder. Br J Psychiatry 192(2):118-123

Philipsen A, Feige B, Hesslinger B et al (2009) Borderline typical symptoms in adult patients with attention deficit/hyperactivity disorder. Atten Defic Hyperact Disord 1(1):11-18

Prada P, Hasler R, Baud P et al (2014) Distinguishing borderline personality disorder from adult attention deficit/hyperactivity disorder: a clinical and dimensional perspective. Psychiatry Res 217(1-2):107-114

Preisig M, Fenton BT, Matthey ML et al (1999) Diagnostic interview for genetic studies (DIGS): inter-rater and test-retest reliability of the French version. Eur Arch Psychiatry Clin Neurosci 249(4):174-179

Ravindran AV, Kennedy SH, O'Donovan MC et al (2008) Osmoticrelease oral system methylphenidate augmentation of antidepressant monotherapy in major depressive disorder: results of a double-blind, randomized, placebo-controlled trial. J Clin Psychiatry 69(1):87-94

Romo L, Legauffre C, Mille S et al (2010) Psychometric properties of the French version of the Wender Utah Rating Scale and Brown's Attention Deficit Disorders Scale for adults. Encephale 36(5):380-389

Shaw P, Stringaris A, Nigg J et al (2014) Emotion dysregulation in attention deficit hyperactivity disorder. Am J Psychiatry 171(3):276-293

Sinita E, Coghill D (2014) The use of stimulant medications for noncore aspects of ADHD and in other disorders. Neuropharmacology $87: 161-172$

Skodol AE, Gunderson JG, Pfohl B et al (2002) The borderline diagnosis I: psychopathology, comorbidity, and personality structure. Biol Psychiatry 51(12):936-950

Spielberger C (1998) State-trait anger expression inventory, research edition. Professional Manual. Psychological Assessment Resources, Odessa, FL

Stoffers JM, Vollm BA, Rucker G et al (2012) Psychological therapies for people with borderline personality disorder. Cochrane Database Syst Rev 8:CD005652

Trivedi MH, Cutler AJ, Richards C et al (2013) A randomized controlled trial of the efficacy and safety of lisdexamfetamine 
dimesylate as augmentation therapy in adults with residual symptoms of major depressive disorder after treatment with escitalopram. J Clin Psychiatry 74(8):802-809

van Dijk FE, Lappenschaar M, Kan CC et al (2012) Symptomatic overlap between attention-deficit/hyperactivity disorder and borderline personality disorder in women: the role of temperament and character traits. Compr Psychiatry 53(1):39-47

van Reekum R, Links PS (1994) N of 1 study: methylphenidate in a patient with borderline personality disorder and attention deficit hyperactivity disorder. Can J Psychiatry 39(3):186-187

Volkow ND, Swanson JM (2013) Clinical practice: adult attention deficit-hyperactivity disorder. N Engl J Med 369(20):1935-1944

Ward MF, Wender PH, Reimherr FW (1993) The Wender Utah Rating Scale: an aid in the retrospective diagnosis of childhood attention deficit hyperactivity disorder. Am J Psychiatry 150(6):885-890
Yeomans FE, Levy KN, Caligor E (2013) Transference-focused psychotherapy. Psychotherapy 50(3):449-453

Zanarini MC (2000) Childhood experiences associated with the development of borderline personality disorder. Psychiatr Clin North Am 23(1):89-101

Zanarini MC, Williams AA, Lewis RE et al (1997) Reported pathological childhood experiences associated with the development of borderline personality disorder. Am J Psychiatry 154(8):1101-1106

Zanarini MC, Frankenburg FR, Dubo ED et al (1998) Axis I comorbidity of borderline personality disorder. Am J Psychiatry 155(12):1733-1739 